\title{
FERTI-MIEUX: une expérimentation sociale pour gérer les risques pour l'environnement en partageant des connaissances scientifiques
}

\author{
FERTI-MIEUX : a social experimentation for managing environmental \\ risks and sharing scientific knowledges
}

par Danielle Lanquetuit, agronome à la Chambre d'Agriculture de Seine-et-Marne*

\footnotetext{
- Elle a présenté en novembre 1998, lors du colloque « Agriculture et Environnement », l'usage du guide FERTI-MIEUX écrit avec Michel Sebillotte et la participation des instances de FERTI-MIEUX-ANDA et des actions locales
}

FERTI-MIEUX is a label conceded to 54 local actions of development which join together water actors, agriculturists and technical advisers in order to reduce pollution risks due to nitrates and to maintain agriculture income. The scientific knowledges are used but there are still uncertainties : it can not be waiting until we know everything before acting.

\section{INTRODUCTION}

FERTI-MIEUX est un label accordé aujourd'hui à 54 actions locales de développement réunissant acteurs de l'eau, agriculteurs et conseillers pour réduire les risques de pollution des eaux par les nitrates et maintenir le revenu net de l'agriculture.

Les actions respectant la démarche ont d'abord un prélabel pour un an, puis un label, renouvelable tous les deux ans. C'est un contrat de confiance avec des améliorations progressives.

\section{D LES PRINCIPES DE FERTI-MIEUX}

Les actions FERTI-MIEUX reposent sur un double partenariat des agriculteurs avec la recherche et les acteurs de l'eau. Cela conduit à mettre au point des méthodes d'évaluation de la réduction des risques, ce qui est très différent de la simple mesure de teneurs en nitrates dans les eaux.

C'est pourquoi la méthode d'évaluation publiée dans le guide FERTI-MIEUX ${ }^{1}$ est un outil indispensable à la négociation sociale. Il s'agit de conduire objectivement les évaluations des modifications de pratiques pour témoigner devant la société de l'efficacité des actions, pour prouver que les pratiques évoluent dans le sens d'une réduction des risques de rejets des nitrates vers les eaux.

1 Lanquetuit D., Sebillotte M., 1997. Protection de l'eau, le guide FERTIMIEUX pour évaluer les modifications de pratiques des agriculteurs, Association Nationale pour le Développement Agricole, Paris, 180 p.
L'opération FERTI-MIEUX, avec le guide pour évaluer les modifications de pratiques des agriculteurs, ouvre la voie à une définition plus précise des politiques de prévention des risques à mener en concertation avec les acteurs locaux.

L'opération FERTI-MIEUX s'engage sur une obligation de moyens : la réduction des risques de pertes de nitrates par l'adoption de pratiques conseillées en fonction des milieux et du climat local. Elle doit pouvoir prouver que l'agriculture est capable de progresser par le conseil.

Le guide est destiné en priorité aux responsables et aux techniciens des actions FERTI-MIEUX, et aussi à tous ceux qui travaillent sous des formes diverses à l'amélioration de l'environnement et au dialogue social.

Un résumé de la méthode a été publié dans les actes du colloque organisé par la Société Hydrotechnique de France en novembre 1998. La présentation de l'expérience FERTIMIEUX était réalisée de façon complémentaire par Jean SALMON, Michel SEBILLOTTE et Danielle LANQUETUIT $^{2}$.

Ce témoignage montre comment les acteurs locaux ont évolué en étant accompagnés par l'organisation nationale et le réseau des actions.

Aujourd'hui les outils d'évaluation mis au point ont un rôle important pour aborder l'environnement sous un angle positif.

2 J. SALMON est agriculteur, président du Comité National de Pilotage de FERTI-MIEUX, M. SEBILLOTTE est professeur d'agronomie, directeur scientifique de la DADP auprès du président de l'INRA et président du Comité Scientifique et Technique de FERTI-MIEUX et D. LANQUETUIT, agronome, a participé au Secrétariat Technique de 1991 à 1995. 
Pour produire notre nourriture, les agriculteurs et les éleveurs ont des savoir-faire basés sur l'agronomie.

Les mystères du cycle de l'azote ont été mis à jour en 1969 avec la méthode du bilan.

20 ans après, en 1989, les Agences de l'Eau ont demandé de faire mieux pour protéger l'eau. Des connaissances anciennes et nouvelles ont été mises à disposition des conseillers, dans des ouvrages de références pratiques :

- orange, tous les dossiers du CORPEN,

- vert le colloque INRA de 1990,

— bleu le guide FERTI-MIEUX paru en 1997.

L'aventure FERTI-MIEUX est une formation, un apprentissage collectif de la gestion des risques, avec les exigences d'une démarche scientifique et la reconnaissance d'un label pour une action de groupe.

Les acteurs du terrain réclamaient une recette pour évaluer l'efficacité des actions. Avec eux et pour eux, FERTIMIEUX a réalisé un guide en 3 étapes pour composer un parcours raisonné et adapté.

Ouvrons ce guide qui décrit trois étapes.

L'étape 1 met en avant un indicateur simple : le solde “apports - exportations" à l'exploitation. Ce calcul, repris pour le suivi de la directive nitrate, est utilisé ici avec deux atouts : l'échantillonnage des exploitations est cohérent avec la diversité des risques et l'interprétation est fondée sur l'agronomie. Cette étape amène à se poser des questions selon le cycle de l'azote et le climat.

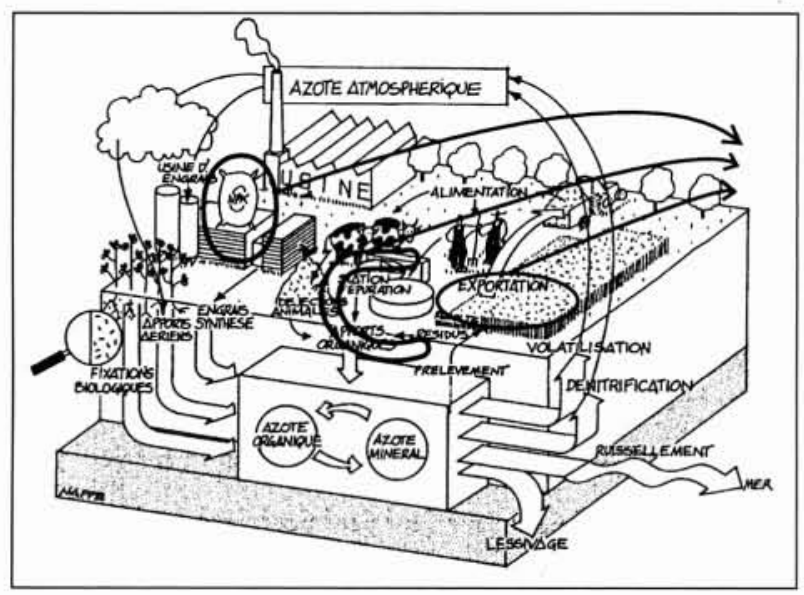

1. Le cycle de l'azote.

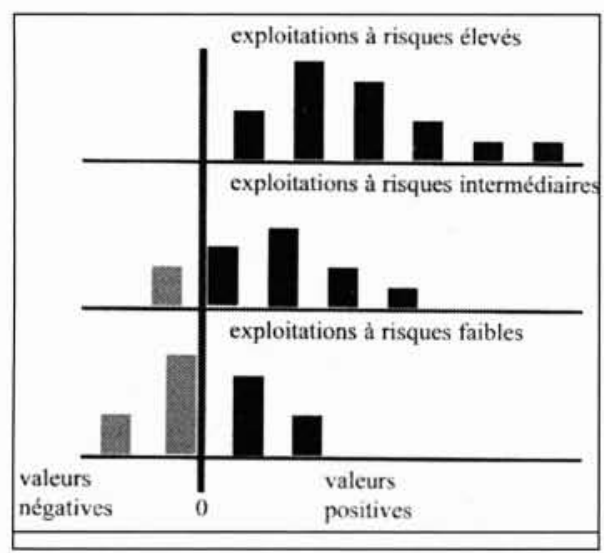

Dans l'étape 2, on raisonne sur tous les indicateurs du cycle de l'azote selon un calendrier pour classer les risques réels en tenant compte du sol et du climat, et des probabilités de risque.

Une pratique peut corriger la vulnérabilité d'un milieu. Cette étape conduit à s'interroger sur l'étendue de chaque pratique.

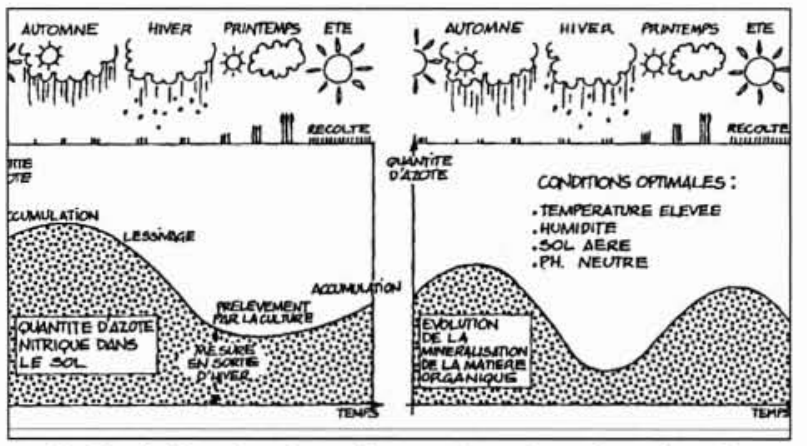

3. Variation des fournitures du sol en fonction de l'époque de l'années et des conditions climatiques.

\begin{tabular}{|c|c|c|c|}
\hline \multicolumn{4}{|c|}{ Étendue des situations à risques } \\
\hline \multirow{2}{*}{$\begin{array}{l}\text { variabilité } \\
\text { interannuelle } \\
\text { des } \\
\text { potentialités } \\
\text { agricoles }\end{array}$} & \multicolumn{3}{|c|}{$\begin{array}{l}\text { Risques de perte hors de porté } \\
\text { des racines les plus profondes } \\
\text { durant les cycles culturaux successifs }\end{array}$} \\
\hline & faibles à nuls & intermédiaires & forts à certain \\
\hline \multirow[b]{2}{*}{ faible } & $\mathbf{A}=$ & $\mathbf{B}=$ & $\mathrm{C}=$ \\
\hline & SAU en ha & SAU en ha & ISAU en ha \\
\hline \multirow{2}{*}{ forte } & $\mathbf{D}=$ & $\mathbf{E}=$ & $\mathbf{F}=$ \\
\hline & SAU en ha & & SAU en ha \\
\hline
\end{tabular}

4. Répartition des surfaces du bassin selon six niveaux de risque.

L'étape 3 engage la réflexion sur la gestion du territoire, donc en proposant des compensations de risques entre parcelles, entre exploitations. On regagne des degrés de liberté car les solutions deviennent plus nombreuses.

Le contrat de confiance peut être autre chose qu'un principe de précaution exigeant une " pollution zéro à tout instant sur chaque parcelle".

On s'interroge sur la localisation des pratiques à risques pour en estimer l'impact selon la circulation de l'eau.

Les cartes sont utiles pour poser les bonnes questions et exprimer différents scénarios de prévention.

Le contrat FERTI-MIEUX actuel est une obligation de moyens sur les pratiques. Et il n'est pas facile de patienter en attendant la réponse de l'eau. Aussi, schémas et cartes aident-ils au dialogue avec les hydrogéologues qui ont déjà participé au diagnostic : les modèles agronomiques décrivent les risques dans les horizons cultivés, mais au-delà, pour prévoir la réponse de l'eau, des connaissances sur les transferts hydrologiques sont nécessaires. Les tableaux de bord " évaluation des modifications de pratiques" sont conçus pour aider les acteurs locaux à patienter ensemble, sans perdre confiance. 


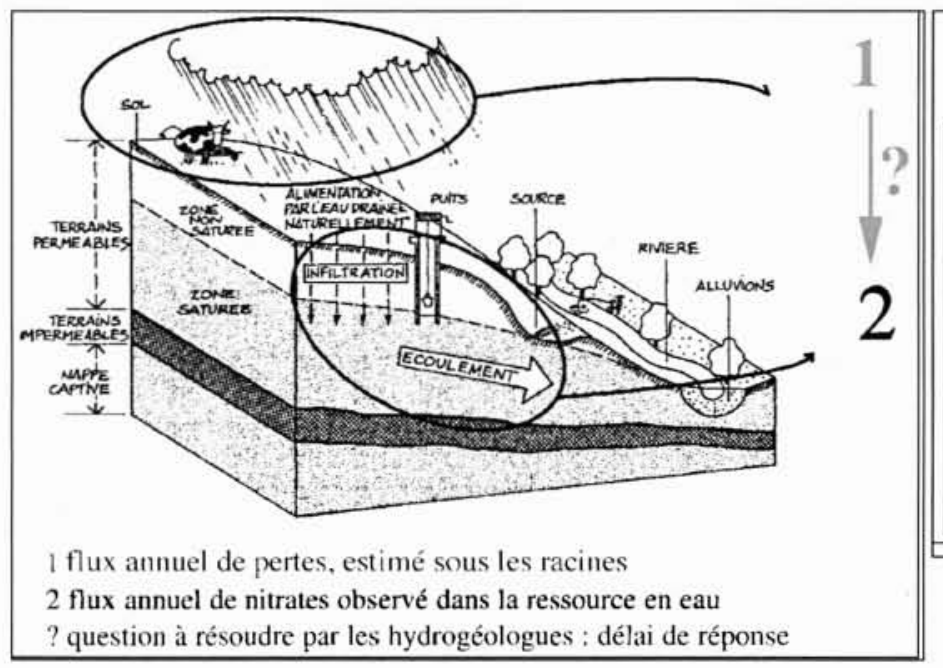

\begin{tabular}{|c|c|c|c|}
\hline \multicolumn{4}{|c|}{$\begin{array}{l}\text { Impact des pratiques moyennes } \\
\text { sur les pertes potentielles }\end{array}$} \\
\hline \multirow{2}{*}{$\begin{array}{l}\text { variabilité } \\
\text { interannuelle } \\
\text { des } \\
\text { potentialités } \\
\text { agricoles }\end{array}$} & \multicolumn{3}{|c|}{$\begin{array}{l}\text { Risques de perte hors de portée } \\
\text { des racines les plus profondes } \\
\text { duram les cycles culturaux suceessifs }\end{array}$} \\
\hline & faibles á nuls & intermediaires & forts à certains \\
\hline \multirow[b]{2}{*}{ faible } & $A=$ & $\mathbf{B}=$ & $C=$ \\
\hline & $\begin{array}{l}\mathrm{kg} \mathrm{N} / \mathrm{ha} \\
x \text { SAU en ha }\end{array}$ & $\begin{array}{l}\mathrm{kg} \mathrm{N} / \mathrm{ha} \\
\mathrm{x} \text { SAU en ha }\end{array}$ & $\begin{array}{l}\mathrm{kg} \mathrm{N} / \mathrm{ha} \\
\mathrm{x} \mathrm{SAU} \text { en ha }\end{array}$ \\
\hline \multirow{2}{*}{ forte } & $\mathbf{D}=$ & $\mathbf{E}=$ & $\mathbf{F}=$ \\
\hline & $\begin{array}{l}\mathrm{kg} \mathrm{N} / \mathrm{ha} \\
\times \mathrm{SAU} \text { en ha }\end{array}$ & $\begin{array}{l}\mathrm{kg} \mathrm{N} / \mathrm{ha} \\
\mathrm{x} \mathrm{SAU} \text { en ha }\end{array}$ & $\begin{array}{l}\mathrm{kg} \mathrm{N} / \mathrm{ha} \\
\mathrm{x} \text { SAU en ha }\end{array}$ \\
\hline
\end{tabular}

6. Outil pour définir des objectifs de qualité collectifs.

\section{Le fonctionnement d'une nappe.}

En choisissant dans les documents orange du CORPEN un modèle de lessivage adapté au sol cultivé, on estime les flux de pertes avec la grille de risque, on interprète au niveau local pour réaliser le tableau de bord accompagnant la carte. Le niveau de maîtrise des risques collectifs devient communicable et scientifiquement acceptable.

Ce label est un contrat de confiance sur la réduction des risques. Mais dans quel délai les teneurs des eaux serontelles aux normes ? Pour le prévoir, on a besoin du savoir des hydrogéologues associés.

\section{III — FERTI-MIEUX DANS LA PRATIQUE}

Et sur le terrain ? Cette matière grise a les couleurs de la vie... Regardons ce qui se passe ici et là...

Un coin de France vu par les agriculteurs... ce n'est ni une carte, ni un paysage, mais ce sont des parcelles cultivées avec leur histoire.

Dans les actions les plus en avance, cette vision est éclairée par les connaissances hydrogéologiques et organisée par les agronomes en cartes d'assolement utilisées pour le diagnostic des pratiques, pour le choix des réseaux de parcelles où seront faites des analyses de sols, de végétaux, des tours de plaine, des enquêtes culturales, pour élaborer et vérifier les conseils.

Des cartes de pronostic de risques sont utiles pour aider aux décisions individuelles.

Sur une carte, on peut montrer l'impact prévisible d'un scénario de modifications de pratiques pour aider aux décisions en groupe...

Outil de pilotage et outil d'évaluation d'impact prennent la même forme pour simplifier le dialogue.

Des cartes par pratiques sont élaborées pour suivre les modifications réalisées, définir les marges de progrès et négocier la répartition des coûts de prévention.

Le regard des agriculteurs et des conseillers change, celui des acteurs de l'eau aussi. En regardant l'eau de manière scientifique et non passionnelle, certains fossés d'incompréhension ont été franchis...

Avec des connaissances partagées, on n'engage pas d'actions médiatiques comme les bandes en herbe sans réfléchir à la gestion du territoire.
En observant l'eau pour mieux comprendre la terre, c'est un retour au tour de plaine agronomique ${ }^{3}$. Avec FERTIMIEUX, les acteurs locaux trouvent un langage commun en utilisant différentes sciences pour comprendre, proposer des scénarios, prévoir leur impact. Ils définissent ensemble les étapes et les délais pour atteindre un objectif collectif.

Sur un bassin versant de Seine et Marne, des " promoteurs de verdure " faisaient l'éloge des bandes en herbe en fond de vallée au bord des rivières, parce que cela se fait ailleurs, parce que cela se voit . Mais ici, c'est sur le plateau drainé que se joue la qualité des eaux de surface. La localisation de bandes en herbe efficaces serait différente : au bord des rûs sur le plateau ou en côteaux. Et $6 \%$ seulement de la surface pourrait être concernée. Une carte est en cours de réalisation pour proposer des scénarios avec estimation d'impact et de coût.

Les esprits sont prêts à réaliser ce diagnostic de terrain, suite au travail engagé pour les nitrates avec la démarche FERTI-MIEUX. Ce sont aussi les questions pressantes sur les produits phytosanitaires qui motivent cette formation mutuelle (agriculteurs, agronomes, prescripteurs, acteurs de l'eau).

\section{IV — LA COMMUNICATION ENTRE LES DIFFÉRENTS ACTEURS}

Le savoir-faire précède le savoir-dire : instaurer la confiance en partageant des savoir-faire, évaluer l'impact des actions avec des preuves, cela permet ensuite de pouvoir dire. Mais communiquer des sciences complexes n'est pas facile.

Parler aux scientifiques, aux agriculteurs, aux acteurs de l'eau, au grand public, aux écoles, savoir dire à tous ces publics, c'est être multilingue...

Pour expliquer le cycle de l'eau aux enfants et à leurs enseignants, c'est l'Agence de l'eau qui a pris les devants, avec les classes d'eau. Différents acteurs de l'eau y participent à présent.

Les questions des gosses qui réfléchissent sur la planète bleue, ça dérange les adultes qui plongent dans ces classes d'eau : “ dis, c'est vrai qu'il ne reste que 500 jours pour sauver la planète ? dis, ça va devenir comme un désert ici, il n'y aura plus d'eau propre? ".

\footnotetext{
${ }^{3}$ travaux publiés en 1969 par Michel Sebillotte
} 
On peut expliquer, rassurer... C'est facile de donner confiance à un gosse, mais ce regard là nous engage à l'action, au-delà des paroles....

Cela engage à expliquer aux adultes comment agir ensemble, sans perdre de temps : la mallette pédagogique est toujours prête, pour aller expliquer ce qui se passe dans les actions locales, en allant à la rencontre des gosses ou des partenaires de l'eau, qui nous obligent à décoder nos langages trop techniques.

Savoir dire, c'est aussi expliquer aux conseillers agricoles ce qu'il faut changer dans leur métier...

Dans le guide FERTI-MIEUX, quatre aquarelles reprennent les temps forts d'une action de prévention, pour mieux s'approprier un changement, s'identifier à des acteurs qui progressent.

Car motiver les prescripteurs demande aussi des précautions... Les anciens qui ont participé à l'augmentation de la production se sont sentis remis en cause, et les nouveaux n'ont pas toujours été clairement encouragés par leurs employeurs-agriculteurs, et malgré le chemin parcouru dans de nombreuses actions locales, et en particulier depuis 1991 avec FERTI-MIEUX, l'environnement n'est pas encore décrit comme un atout dans toutes les conversations quotidiennes.

Pour accompagner cette évolution, deux sessions de formation ont été réalisées pendant la rédaction du guide. Elles ont rassemblé une cinquantaine de conseillers parmi les 1500 participants aux équipes techniques des 54 actions labellisées sur tout le territoire.

Apprendre à gérer l'espace pour gagner des marges de liberté, c'est mettre au point de nouveaux conseils et partager les expériences de diverses régions.

En sept ans, cette démarche de gestion collective d'un territoire a engagé 25000 exploitations agricoles en France dans la gestion de ressources en eau stratégiques pour un grand nombre d'habitants. Ils s'entraînent à décider en groupe pour répartir les efforts de prévention, sans handicaper la performance économique de leurs entreprises, et en utilisant au mieux les financements locaux.

Trois générations participent. La génération qui a les cheveux blancs transmet un savoir produire pour nourrir. Si certains freinent la prise en compte de l'environnement, au nom de nécessités économiques, d'autres veulent aller de l'avant plus vite, pour faire de l'environnement un atout, avec d'autant plus de conviction quand ils sont maires de leurs communes...

Agriculteurs, éleveurs, scientifiques, conseillers ont mille visages. Vu de Seine et Marne, ceux qui sont partis les premiers s'appellent Charles Philippe à Doue, Jacques Michel sur la Voulzie, Jean Salmon et Michel Sebillotte avec FERTI-MIEUX... C'est avec eux que l'équipe de la Chambre d'Agriculture a participé à cette évolution du conseil agricole.

Avec eux, les acteurs de l'eau ont engagé un dialogue franc, et l'hydrogéologie associée à l'agronomie permet de redécouvrir la terre. Nous apprenons à protéger l'eau. Ceux qui sont venus les premiers à la rencontre de l'agriculture pour dialoguer en Seine et Marne s'appellent René Delouvée et Véronique Jovy pour l'Agence de l'Eau, Jean Michel Laya et Gilbert Fournol pour la SAGEP au niveau local, avec les 25 membres du CST FM au niveau national, et sur le terrain aujourd'hui de nouveaux acteurs qui nous font avancer à grands pas : Claude Meignien, hydrogéologue, Nils Fauchon de la Compagnie Générale des Eaux (Vivendi aujourd'hui)...
La génération des " agri-managers " ne veut pas renoncer à produire en quantité, car le marché mondial bouge, et il fait faim sur la planète.

Pour eux, intégrer les outils de gestion FERTI-MIEUX ne pose pas de problème en région de grandes cultures car on peut doser les efforts et préserver la qualité des récoltes.

En région d'élevage, les modifications de pratiques sont plus complexes lorsque les surfaces d'épandage manquent. Mais des solutions de compostage s'organisent, et le retour à la production d'herbe remplace parfois la rotation ray-grassmaîs pour des raisons d'organisation du travail. C'est la preuve que des motivations économiques peuvent aussi réduire les risques pour l'eau.

Cette génération est prise entre deux feux et s'interroge : l'environnement restera-t-il une contrainte ou peut-il devenir un atout ? Peut-on vraiment faire confiance quand le principe de précaution conduit à des réglementations en chaîne ?

Quant à la génération qui s'installe, elle sait qu'elle doit réussir un double pari : produire à manger et à boire... Elle se passionne aussi pour l'agriculture de précision, et imagine plus facilement la gestion de bassins de production et de bassins versants avec des informations relayées par satellites...

La gestion économique à la parcelle et la gestion du territoire utilisant des informations communes, de nouvelles solutions de traitement des données semblent possibles, en équipant par exemple les coopératives avec des systèmes d'information géographique, les tableaux de bords sur l'impact des pratiques seraient renseignés par bassin de production. Des tests sont en cours.

Trois générations dialoguent avec les outils FERTIMIEUX... pour une gestion du territoire avec un double partenariat permanent : la recherche et les acteurs de l'eau sont partie prenante. C'est un contrat de confiance qui intéresse les acteurs économiques agricoles.

\section{V घ DU CÔTÉ DES COOPÉRATIVES AGRICOLES}

Regardons du côté des coopératives, en ouvrant une revue interne.

La revue Agro-performance publiée par la coopération agricole montre bien que la fertilisation azotée est un enjeu pour l'agriculture : les coopératives contribuent fortement au développement des outils de conseil à la parcelle pour plusieurs raisons. Leurs responsables sont des agriculteurs engagés à la fois dans la sécurité alimentaire (quantité et qualité) et dans la protection de l'environnement à travers FERTIMIEUX (national et local).

Multiplier les analyses de sols et de végétaux est aussi un atout économique pour une entreprise de service. Le conseil intègre donc aujourd'hui l'environnement comme un atout économique.

La préface, rédigée par Michel Sebillotte, montre le chemin à parcourir pour aller du conseil à la parcelle à la gestion collective des risques sur un territoire. Une piste est proposée, sous forme de question: “ pourquoi ne pas utiliser la nouvelle loi d'orientation et les contrats territoriaux d'exploitations (C.T.E.) pour combiner l'esprit de la réglementation et la démarche de FERTI-MIEUX comme source de certification ?".

Oui, des idées germent... En septembre 97, un producteur de fruits engagé dans la certification COVAPI évoquait l'idée d'un " comité d'éthique " associant des consommateurs à la 
vérification de la qualité... Cela a fait un peu peur, mais il ne s'agissait pas de mettre des consommateurs aux conseils d'administration des coopératives agricoles pour des décisions économiques. Depuis, des groupements de développement ont entrepris un dialogue avec des consommateurs pour définir des cahiers des charges sur la qualité des récoltes.

Quelques mois plus tard, un responsable de groupe de développement décrivait les actions pilotes comme des “ coopératives d'idées "... Ce mot correspond à des expériences positives, et ne fait pas peur.

Quelques mois ont encore passé, et la notion de C.T.E. (Contrat Territorial d'Exploitation) a été lancée... 70 dépts réfléchissent...

Les Comités de Pilotage des actions labellisées pourrontils fonctionner comme des " Coopératives Territoriales pour l'Eau ", et être reconnus garants du bon usage de l'argent public de C.T.E. par exploitations ? L'action collective précédant ici les initiatives individuelles, il est plus facile de savoir où agir pour un impact maximum.

Les réflexions s'engagent, sans préjuger de la faisabilité, ni des positions politiques professionnelles régionales, liées aux conditions de financement de la loi d'orientation agricole.

Peut-on espérer que le temps passé sur le terrain par les pionniers, les équipements acquis, et tout le travail en commun permette de pérenniser cette " gestion coopérative des risques pour l'eau" si elle correspond aux volontés locales?

Beaucoup a été fait pour permettre cette possibilité, mais attention, le terrain se vide... En effet, les agriculteurs ont moins de temps, et des conseillers sont rentrés au bureau pour remplir les papiers réglementaires, et des hydrogéologues recopient des principes de précaution pour les D.U.P. de captage sans retourner sur le terrain...

Trouver un équilibre entre le temps des contrôles induits par les réglements et les actions innovantes n'est pas simple en ce moment.
Il faut tenir sur le terrain. Les équipes de conseillers auront-elles assez de temps pour " retourner aux champs"?

Ce retour aux champs cultivés est nécessaire pour utiliser les connaissances agronomiques avec les nouveaux moyens d'observation et de communication, comme le retour aux champs captants est nécessaire pour observer et pour comprendre l'eau.

Nous avons besoin des chercheurs sur le terrain pour réunir savoir faire et savoir dire, et définir les bonnes priorités économiques et écologiques.

\section{VI $\square$ CONCLUSION}

Au départ, FERTI-MIEUX a été perçu comme une action de sensibilisation des agriculteurs. Il est visible aujourd'hui qu'il s'agit d'un apprentissage de gestion collective du territoire.

On parle beaucoup des modifications de pratiques des agriculteurs; mais n'oublions pas que les prescripteurs doivent d'abord changer leurs conseils.

Il est visible qu'il s'agit aussi de formation mutuelle : des acteurs de l'eau comprennent mieux l'agriculture, des agriculteurs intègrent la protection de l'eau, et des chercheurs sont engagés dans l'action, d'une manière plus forte qu'un bureau d'étude.

Ce travail d'équipe est un nouveau langage. Parfois, nous le nommons entre nous "Espéranteau"...

Pour la prévention nitrate, 54 lieux existent, où la formation a précédé la réglementation nouvelle et produit des solutions que l'on espère durables.

Ces savoir-faire contribuent aussi à la réflexion pour les produits phytosanitaires, même si les risques sont d'un autre niveau.

Le guide FERTI-MIEUX est une invitation à l'action locale. 